\title{
Risiken und Nebenwirkungen einer naiv evidenzbasierten Grundschulpädagogik zu Inklusion und Partizipation
}

\author{
Kathrin Müller • Agnes Pfrang
}

Eingegangen: 1. November 2020 / Angenommen: 12. März 2021 / Online publiziert: 9. Juli 2021 (C) Der/die Autor(en) 2021

Zusammenfassung Der Beitrag verfolgt das Ziel, Fragen nach den Potenzialen einer rein evidenzbasierten Grundschulpädagogik für Inklusion und Partizipation zu erörtern. Dazu werden in einem ersten Schritt Inklusion und Partizipation als mehrdimensionale soziale Prozesse beschrieben, bevor in einem zweiten Schritt auf Risiken und Nebenwirkungen einer naiv evidenzbasierten Grundschulpädagogik und auf die Notwendigkeit eines multiparadigmatischen Zugangs zur Grundschulpädagogik eingegangen wird.

Schlüsselwörter Inklusion · Partizipation · Evidenzbasierte Grundschulforschung · Evidenzbasierte Grundschulpädagogik

Jun. Prof. Dr. Kathrin Müller (凷

Institut für Erziehungswissenschaft, Arbeitsbereich Schulpädagogik, Pädagogische Hochschule Ludwigsburg, Reuteallee 46, 71634 Ludwigsburg, Deutschland

E-Mail: k.mueller@ph-ludwigsburg.de

Prof. Dr. Agnes Pfrang

Erziehungswissenschaftliche Fakultät, Fachgebiet Grundschulpädagogik und Kindheitsforschung, Universität Erfurt, Nordhäuserstr. 63, 99089 Erfurt, Deutschland

E-Mail: agnes.pfrang@uni-erfurt.de 


\section{Risks and side effects of a naive evidence-based primary school pedagogy on inclusion and participation}

Abstract The article aims to discuss the potentials of a solely evidence-based primary school pedagogy for inclusion and participation. Regarding to this we describe inclusion and participation as multidimensional social processes, firstly. Secondly, we discuss the risks and side effects of a naive evidence-based primary school pedagogy and the necessity of a multiparadigmatic approach to primary school pedagogy.

Keywords Inclusion - Participation - Evidence-based primary-school studies · Evidence-based primary-school pedagogy

\section{Einleitung}

Historisch betrachtet verstärkte sich die „Evidenzbasierung“ in der Pädagogik im Anschluss an PISA, IGLU und anderen Bildungsvergleichsstudien. Insbesondere mit dem US-amerikanischen „No-child-left-behind-Gesetz“ (2002) erhielt das Postulat der Evidenzbasierung in der Pädagogik eine neue Dimension. Die datengestützte und Output-orientierte Steuerung des Bildungssystems wurde zum Programm der Bildungspolitik. Da es spätestens seit der UN-Behindertenkonvention (UN-BRK) ein erklärtes politisches Ziel ist, allen Kindern und Jugendlichen gleiche Bildungschancen und uneingeschränkte Teilhabe an allen Lebensbereichen - also auch in der Grundschule - zu ermöglichen, lässt der Wunsch nach einer evidenzbasierten Gestaltung von Inklusion und Partizipation in der Grundschulpädagogik nicht lange auf sich warten. Hier bedarf es aber einer grundschulspezifischen Reflexion über die Dynamiken, die aus der Verbindung von Inklusion und Partizipation mit Evidenzbasierung einhergehen.

Im Beitrag wird die Frage diskutiert, inwiefern sich ein evidenzbasierter Zugang zu Inklusion und Partizipation als ein wissenschaftliches, praktisches und politisches Handlungsfeld überhaupt für die Grundschulpädagogik eignet. Hierfür verorten wir in einem ersten Schritt Inklusion und Partizipation als eine reziproke Aufgabe von Gesellschaft und Individuum, die sich im normorientierten sozialen Miteinander von Gemeinschaft und Individuum vollzieht. Dann wenden wir uns der Evidenzbasierung zu, um aufzuzeigen, was diese bezweckt. Anhand dessen lassen sich die Problemstellen benennen, die eine naiv evidenzbasierte Grundschulpädagogik zu Inklusion und Partizipation hinterlässt. Da Evidenzbasierung ihr Augenmerk besonders auf die Qualitätsverbesserung der Praxis richtet, erscheinen das Verständnis des Verhältnisses von Wissenschaft und grundschulpädagogischer inklusiver Praxis, der Glaube, Inklusion sei steuerbar, ein technokratisches Verständnis von Lernen sowie das Verhältnis von Wissenschaft und Politik bedenkenswert. 


\section{Inklusion und Partizipation - Lesarten vieldiskutierter Begriffe}

Inklusion und Partizipation sind in den aktuellen sozial- und geisteswissenschaftlichen Diskursen vielgebrauchte, aber dennoch unscharf verwendete Begriffe. Die Diskussionen im Kontext von Bildung und Schule fokussieren dabei gerechtigkeitstheoretische, menschenrechtliche, sozialkritische, anthropologische und demokratische Überlegungen. Eine gerechtigkeitstheoretische Verortung erfolgt dabei meist über die UN-Behindertenrechtskonvention (UN-BRK 2006, Art 24). Inklusion und Partizipation werden in diesen Auseinandersetzungen mit dem Grad an Mitbestimmungs- und Teilhabemöglichkeiten gleichgesetzt, die Heranwachsenden z.B. in Schule und Unterricht gewährt werden. Aufgrund der historischen Entwicklung der UN-BRK ist eine sozialkritische Perspektive den gerechtigkeitstheoretischen $\mathrm{Zu}$ gängen zu Inklusion und Partizipation immanent, in der Behinderung auch in ihrer systemischen Bedingtheit gelesen wird. Nach K. Müller (2018) ist die UN-BRK als eine Vertiefung und Erweiterung des Verständnisses von sozialer Gerechtigkeit zu werten. „Um soziale Gerechtigkeit herzustellen, muss auch die Gesellschaft in den Blick genommen werden. Sie hat [...] dafür zu sorgen, dass sich Menschen auf Augenhöhe begegnen können und gleichwertig sowie gleichberechtig agieren können, auch wenn sie von Marginalisierungsprozessen bedroht sind“ (S. 97). Zusammenfassend rekurriert dieser Diskurs auf ein Verständnis von inklusiver Pädagogik, das Biewer (2010) wie folgt beschreibt: Inklusive Pädagogik bezeichnet „Theorien zur Bildung, Erziehung und Entwicklung, die Etikettierungen und Klassifizierungen ablehnen, ihren Ausgang von den Rechten vulnerabler und marginalisierter Menschen nehmen, für deren Partizipation in allen Lebensbereichen plädieren und auf eine strukturelle Veränderung der regulären Institutionen zielen, um der Verschiedenheit der Voraussetzungen und Bedürfnisse aller Nutzer/innen gerecht zu werden“ (Biewer 2010, S. 193). Inklusion erfährt dadurch eine stark normative Orientierung für die Gestaltung unseres Miteinanders. Das Konstrukt Gerechtigkeit wird auf den Aspekt der Partizipationsmöglichkeiten im Sinne eines Beteiligtseins bezogen. Diese Möglichkeiten sind systemisch bereitzuhalten. In diesem Sinn werden im inklusiven Diskurs Teilhabe und Partizipation auch immer wieder synonym verwendet oder miteinander verquickt (Danz 2020).

Doch neben diesem stark normativ-soziologisch geprägten, intransitiven Verständnis von Inklusion, Partizipation und Teilhabe kann dieses auch transitiv konnotiert und kulturanthropologisch im Sinne von Teilhaben verstanden werden. Dadurch ergeben sich vielfältige weitere Facetten für den pädagogischen Diskurs zu Inklusion, Partizipation und Teilhabe. So arbeitet z. B. Dietrich (2017, S. 29ff.) zu „Teilhabe" heraus, dass Teilhabe unterschiedliche Dimensionen, Formen und Qualitäten haben könne. „Durch diese Mehrdimensionalität kann Teilhabe nur als dynamischer Begriff verstanden werden, da sich im Laufe der Zeit Formen und Qualitäten, Widersprüchlichkeiten und Ergänzungen, prekäre und stabile Modalitäten von Teilhabe immer wieder verändern können“ (ebd., S. 30). An diese Überlegungen anknüpfend wird gerade die transitive Bedeutung von Teilhabe als individuelle, volitionale Voraussetzung von Inklusion und Partizipation pädagogisch bedeutsam. „Wird aber in der Teilhabe an Bildung nur erst die Vorbereitungsinstanz für gesellschaftliche Teilhabe gesehen, verschwindet in dieser Doppelstruktur eine Thematisierung der Frage 
nach Wählbarkeit und Freiwilligkeit [...] Die sogenannte Teilhabegerechtigkeit kann damit auch als Zwang aufgefasst werden, sich an einer Mehrheits- und Dominanzkultur (Rommelspacher 1995; 2002) beteiligen zu müssen ohne jemals im Leben die kulturellen, symbolischen sowie ökonomischen Kräfte aufbauen zu können, sich für oder gegen die Teilnahme zu entscheiden“ (ebd.).

Bindet man diese Gedanken an gerechtigkeitstheoretisch normative Ideen zurück, so wird ein Perspektivwechsel von einer rein positiven Kontierung von Inklusion und Partizipation hin zu einem hochkomplexen Gegenstandfeld deutlich, das sich im Spannungsfeld von Gleichheit und Freiheit normorientiert vollzieht. Als dynamisches Handlungsfeld unterliegen Teilhabe, Inklusion und Partizipation in ihrer Mehrdimensionalität situations- und personenabhängigen Brüchen und Widersprüchen sowie Qualitäten und Formen. Dabei können Inklusion und Partizipation sowohl transitiv als auch intransitiv gelesen werden. Sie sind durch die Interaktion zwischen der persönlich psychisch-körperlichen Verfasstheit eines jeden Menschen und den auffindbaren sozialen Strukturen determiniert (vgl. dazu auch Schwab 2016, S. 128). Inklusion und Partizipation werden gemäß diesen Überlegungen zu einer reziproken Aufgabe von Gesellschaft und Individuum und vollziehen sich in einem normorientierten sozialen Miteinander von Gemeinschaft und Individuum. Gerade durch ihre kollektive Bedingtheit werden sie in ihren Ambivalenzen und Antagonismen zum pädagogischen, politischen sowie wissenschaftlichen Handlungsfeld und damit eine Herausforderung für grundschulpädagogische Forschung. Hier stellt sich die Frage, ob ein rein evidenzbasierter Zugang zum Feld diese Komplexität überhaupt untersuchen und wiedergeben kann.

\section{Evidenzbasierung - Was heißt das?}

Die um den Millenniumswechsel lauter werdende Forderung nach evidenzbasierter Forschung und Praxis für und an Schulen resultierte aus der grundsätzlichen Kritik an der Qualität der Bildungsforschung. Der nationale und internationale Konsens der Kritik bestand darin, dass die bis Dato bestehende Forschung kaum generalisierbare und reliable Ergebnisse geliefert habe. Für den Transfer wissenschaftlicher Erkenntnisse in die Praxis und für einen Nutzen derselben für Praktikerinnen und Praktiker sei bisherige Forschung methodisch unzureichend und es würde so kaum relevantes Wissen kumuliert (Tooely und Darby 1998). Demzufolge gründen Handlungen und Entscheidungen von Politikern und von pädagogisch Praktizierenden nur unzureichend auf Forschungsbefunden (Schrader 2014). Aus dieser Kritik resultierte die Forderung nach experimentell gestützten kausalen Erklärungen der Effektivität pädagogischer Programme. Damit wird das Ziel verfolgt, die Kluft zwischen Forschung, Praxis und Theorie zu schließen. Nach Tenorth (2014, S. 8) wurde mit dem „No child left behind-Gesetz“ nicht nur ein bildungspolitisches Programm formuliert, sondern gleichzeitig definiert, was unter wissenschaftlicher Forschung zu verstehen sei: „Akzeptable Wissenschaft, das bedeutet dann, [...], im Allgemeinen eine Art von Forschung, in der die Anwendung von rigiden, systematischen und objektiven Verfahren regiert, um reliables und valides Wissen zu generieren, das nicht nur theoretische Qualität hat, sondern zugleich auch relevant ist für pädago- 
gische Praxis und bildungspolitische Programme und Veränderungsstrategien. Als Teilmenge dieser allgemeinen Erwartungen [...] wird dann ein Forschungstypus [...] unterschieden, der schärfere Anforderungen stellt und seit jeher als Modell und Standard der evidenzbasierten Forschung gilt. Forschung bedeutet hier eine ganz spezifische Erkenntnispraxis, ausgezeichnet vor allem dadurch, dass neben den üblichen Gütekriterien - der methodischen und theoretisch strukturierten, hypothesenprüfenden Organisation der Arbeit sowie der üblichen Gütekriterien reliabler und valider Daten - auch ganz bestimmte Designerwartungen und verschärfte Prüfkriterien formuliert werden [...]." Es geht also um den wissenschaftlichen Nachweis, dass pädagogische Programme, Interventionen und Maßnahmen wirksam sind. „Das Hauptanliegen evidenzbasierter Pädagogik liegt in der Optimierung pädagogischer Interventionen und Programme durch methodisch streng kontrolliertes empirisches Wissen über deren Wirksamkeit. Evidenzbasierte Pädagogik sollte nicht mit empirischer Bildungsforschung gleichgesetzt werden“ (Herzog 2019, S. 21). Die zentrale Grundlage ist hierfür das Programm der evidenzbasierten Pädagogik mit den drei wesentlichen Bereichen evidenzbasierter Praxis, evidenzbasierter Forschung und evidenzbasierter Politik. Deshalb bemerkt Herzog (2019) auch kritisch mit Blick auf Wissenschaft und Forschung: „Der Begriff der Evidenzbasierung [sollte] nicht auf Wissenschaft und Forschung, sondern auf Politik und Praxis bezogen werden" (Herzog 2019, S. 23).

Evidenzbasierung geht von der Grundidee aus, dass das Bildungswesen auf Basis evidenter Daten politisch effektiv und effizient gesteuert werden könne. Auch beispielsweise die UN-BRK ist eine politische Zielvorgabe zu Inklusion und Partizipation zu deren Umsetzung sich die Vertragsstaaten verpflichten, so dass entsprechende Aktionspläne seitens der Politik entwickelt wurden (z.B. Aktionsplan der Landesregierung zur Umsetzung der UN-BRK in Baden-Württemberg, Ministerium für Soziales und Integration (2015)). Aufgabe der Forschung wäre es hierbei, Handlungs-, Entscheidungs- und Steuerungswissen zur Verfügung zu stellen. Der Fokus liegt v.a. auf der engen Verknüpfung zwischen Forschung und Steuerung, die dem Begriff Evidenz eine gesellschaftliche Funktion gibt. Tenorth (2014, S. 8) thematisiert in diesem Zusammenhang die Gefahr, dass entsprechend die staatliche Förderpolitik selbst nur Projekte fördern könnte, die entweder entsprechendes Steuerungswissen produzieren oder über Forschung ermittelte Effizienzkriterien in die pädagogische Praxis implementieren wollen. Untermauert wird diese Befürchtung durch die Tatsache, dass mit dem Evidenzparadigma gleichzeitig Kriterien von Wissenschaftlichkeit (NCLB-Gesetz) bzw. ein bestimmtes methodologisches Vorgehen postuliert werden. Weil aber gleichzeitig für die Durchführung von Forschung seitens der Universitäten dringend Drittmittel benötigt und entsprechend eingeworben werden müssen, könnte auf diesem Wege in interne Strukturen der Erziehungswissenschaft eingegriffen werden. Diesem Argumentationsstrang folgend gibt Radtke (2013, S. 13) zu bedenken, dass die großzügige Finanzierung der „Hebel“ für eine „empirischen Bildungsforschung" sei, die im direkten Auftrag der Politik agiere. Aus diesem Blickwinkel betrachtet erscheint es so, als ob (evidenzbasierte) empirische Forschung zum Dienstleister der Bildungspolitik werde (Buchhaas-Birkholz 2009, S. 27) und als „,Zuverlässige[r] Lieferant administrativ verwertbaren Wissens“ (Radtke 2013, S. 13) fungiere. Dieser Argumentation folgend wird von Forschung 
die Erfüllung einer technischen Aufgabe im Rahmen evidenzbasierter Forschung verlangt, die nur einen Ausschnitt der grundschulpädagogischen Forschung zu Inklusion und Partizipation abdecken kann und somit gleichzeitig andere, das komplexe Forschungsfeld betreffende Teilbereiche, unbeachtet ließe. In Konsequenz muss sich die Grundschulpädagogik als Fach die Bedeutung ihres multiparadigmatischen Zugangs immer wieder bewusst machen und sich die Frage stellen, wie sie sich eben diesen Zugang zum Feld als wissenschaftliche Disziplin bewahrt. Damit sich hier nicht unterschiedliche Interessenslagen vermischen, bedarf es für die Umsetzung evidenzbasierte (An-)Forderungen an Forschung und Praxis gerade im Kontext von Inklusion und Partizipation einer kritischen Rückbesinnung auf das Verhältnis von grundschulpädagogischer Wissenschaft, Praxis und Politik. Denn wenn es, wie beschriebenen, eines der Hauptanliegen von Evidenzbasierung ist, wissenschaftliche Nachweise für die Wirksamkeit inklusionspädagogischer Programme, Interventionen und Maßnahmen zu liefern, um dann auf Basis dieser Nachweise die Implementierung von Inklusion und Partizipation politisch besser steuern zu können, muss sich die Grundschulpädagogik mit der Frage auseinandersetzen, ob sie dies „kann“ und auch ,will“.

\section{Evidenzbasierte Grundschulpädagogik zu Inklusion und Partizipation - ein Holzweg?}

\subsection{Sind Inklusion und Partizipation steuerbar?}

In der schulischen Organisations- und Governanceforschung wird u. a. gefragt, wie wissenschaftliches Wissen in der schulischen Praxis Wirksamkeit entfalten kann. Organisationen unterliegen in ihrer Entwicklung umfassenden Eigendynamiken und Einzelorganisationen könnten als sinngenerierende Systeme (Weick 1976) betrachtet werden: So betont z. B. Fend (2006), dass Einzelschulen Vorgaben rekontextualisieren. Diese Effekte könnten noch durch die politischen Bemühungen um Deregulierungsprozesse in Schulen verstärkt werden, so dass Altrichter und Maag Merki (2010) für die Reform der Einzelschulen sogar von einer Krise der Außensteuerung sprechen. $\mathrm{Ob}$ eine evidenzbasierte Grundschulpädagogik nun tatsächlich einen Beitrag zur Steuerung einer inklusiven Grundschule zu leisten vermag, ist deshalb fraglich.

Evidenzbasierte Ansätze in der Forschung können durch ihr standardisiertes Vorgehen immer dann Erkenntnisse für die Grundschulpädagogik liefern, wenn z.B. „Ist-Zustände“ an Schulen (z.B. zur Praktizierung außerunterrichtlicher Angebote an Schulen, STEG-Studie 2019) oder im Unterricht (z. B. zur Wirkung von PeerMonitoring in Hausaufgabenbetreuung bzw. Lernzeiten, STEG-Studie 2019) überprüft werden. Grundsätzlich ist der Nutzen für die Schulpraxis aber aufgrund der „sektorialen“ Bedeutung evidenbasierter Untersuchungen nur eingeschränkt. Das Bild, das eine evidenzbasierte Bildungsforschung von der grundschulpädagogischen Wirklichkeit zeichnen kann, ist auf Annäherungen und Vereinfachungen angewiesen, da es kaum zu erforschen ist, welche Ursachen mit welchen Wirkungen in welchen Beziehungen stehen. Zusätzlich weisen aktuelle Studien aus der Transfer- 
forschung (z. B. Zlatkin-Troitschanskava et al. 2012; Dormann et al. 2016) im Bildungsbereich darauf hin, dass evidenzbasierte Reformstrategien einer Top-DownLogik folgen, wenn sie allein der Wissenschaft zugestehen, wirksames Wissen zu generieren, welches anschließend in der Praxis umgesetzt werden soll. Eine von der Praxis ausgehende Weiterentwicklung bspw. von Schule und Unterricht wird dabei eher als „unerwünschte Entwicklung“ betrachtet (Gräsel 2010, S. 15). Somit tendiert ein evidenzbasierter Ansatz dazu, die pädagogische Praxis aus dem Prozess der Wissensproduktion auszuschalten. So kann eine evidenzbasierte Pädagogik buttomup Bewegungen, die gerade für Inklusion und Partizipation historisch bedeutsam sind, nicht in den Wissenschaftsprozess adäquat einbinden. Beispielhaft sind hier die Behindertenbewegungen (Schönwiese 2016) oder die Entwicklung der ersten Modellversuche zur gemeinsamen Beschulung von Kindern mit und ohne Behinderung zu nennen. Letztere gingen vor allem auf Einzelinitiativen von Eltern und Lehrpersonen zurück und liefern wertvolle Kenntnisse für die Grundschulpädagogik zu Inklusion und Partizipation, wie beispielsweise F.J. Müller (2018) zusammenfasst. Die Forderung allen Menschen gleichermaßen Teilhabe an Bildung zu ermöglichen, führte zuerst zu Veränderungen in der Schulpraxis und erst im unmittelbaren Zusammenhang damit entstanden erste Forschungsbestrebungen. Diese steht jetzt einem komplexen und heterogenen Praxisfeld gegenüber, das kaum von einheitlichen Modellen oder Konzepten geprägt ist. Rahmenbedingungen von Schule und Unterricht, Unterrichtsmaterialien, -medien und -inhalte, Diagnose- und Förderprogramme, aber auch Peer-Learning oder Teamteaching rückten im Kontext von Inklusion in den Fokus der Forschung. Aufgrund der Komplexität und der damit verbundenen Vielseitigkeit der Ansprüche einer inklusiven Beschulung erscheint es zumindest bedenkenswert, ob eine rein evidenzbasierte Forschung, die dazu tendiert, die pädagogische Praxis aus dem Prozess der Wissensproduktion auszuschließen, dem Anspruch nachkommen kann, nicht nur Wissen über, sondern auch Wissen für die Schul- und Unterrichtspraxis zu generieren. Lohnenswert erscheint es an dieser Stelle, einen Blick auf grundschulpädagogische Konzepte zu werfen, die überwiegend nicht dem Paradigma der Evidenzbasierung folgen, deren Ertrag für die inklusive Schul- und Unterrichtspraxis aber Anerkennung verdient. Exemplarisch sind hier die Leistungen der Grundschulforschung im Bereich einer „Pädagogik der Vielfalt““ (z.B. Prengel 2006) bzw. dem Umgang mit „Diversität“ (z. B. Hildebrandt und Maischatz 2020), der Schulbegleitforschung (z. B. Betz et al. 2019; Wiesemann und Schreyer 2008), der inklusionsorientierten Sachunterrichtsdidaktik (z.B. Pech et al., 2019; Gebauer und Simon, 2012) oder der Forschung zur Rekonstruktion individueller Lernwege (z.B. Leßmann 2020; Pape 2016) zu nennen. V.a. in den letzten Jahren - verbunden mit fachbezogenen Inklusionsdebatten - hat sich ein relativ breiter Diskurs zu Partizipation von Lernenden und der Demokratisierung von (Grundschul-)Unterricht entwickelt (z. B. Boban und Hinz 2019). Dies steht in einem engen Zusammenhang mit Entwicklungen im Bereich der allgemeinen Inklusionsforschung, in deren Kontext neben Fragen der Teilhabe zunehmend auch Fragen der Partizipation und Demokratisierung Berücksichtigung finden (z. B. Kruschel 2017). Damit setzt sich, gemäß des menschenrechtlichen und ethischen, u.a. anerkennungstheoretisch begründeten Fundamenten inklusiven Unterrichts (z.B. Moser 2019), ein Verständnis inklusiven Unterrichts durch, für das 
maßgeblich ist, dass „Individualisierung [...] keine machtvoll durchgesetzte didaktische Maßnahme im Sinne einer strukturell forcierten Direktive, sondern ein demokratischer Prozess“ ist, der ,in pädagogischer Beziehung zwischen verantwortlichen Erwachsenen und Kindern, also zwischen Personen und unter dem Primat der Relation" (Seitz 2020, o. S.) stattfindet. Hierbei sind die sachbezogene und die soziale Dimension von Bildung miteinzubeziehen (Götz et al. 2019, S. 18). Letztere werden in der evidenzbasierten Forschung aufgrund eines technokratischen Verständnisses von Lernen jedoch kaum berücksichtigt.

\subsection{Inklusion als pädagogische Technik?}

Auch das an das aus dem anglo-amerikanischen Sprachbereich entlehnte „evidencebased-learning" (EBL) angelehnte Verständnis von Evidenz, erscheint im Kontext von Inklusion und Partizipation diskussionswürdig. Mit „Evidenz“ wird hier „die Überprüfung/Überprüfbarkeit von Lerneffekten“ verstanden, „,d.h. von pädagogischen Professionellen [wird] eine kontinuierliche Kontrolle der Lerneffekte und in diesem Zusammenhang - falls notwendig - eine Korrektur des methodischen Vorgehens - entlang passender, evidenzbasierter Forschungsergebnisse“ (Rödler 2013, S. 381) gefordert. Die dieser evidenzbasierten Pädagogik zugrundeliegende Förderphilosophie setzt auf eine Standardisierung der Fördermaßnahmen, die zugleich eine Normierung der Lern- und Verhaltensprobleme bedingt. Diese Normierung des Lernund Sozialverhaltens widerspricht allerdings der Heterogenität und Individualität der Lernenden (Uphoff 2009, S. 75) und ist gerade im Hinblick auf Inklusion und Partizipation als kritisch zu bewerten. Zusätzlich drängt sich hier die Frage auf, ob ein solches Vorgehen nicht vielmehr das Denken in zwei Gruppen verschärft, statt es zu vermindern. Nämlich erstens in die Gruppe derer, die normorientiert Lernen können und zweitens in die Gruppe der Anderen, die Unterstützung brauchen. Besonders deutlich wird dies, wenn die eruierten Wirkungen z.B. für Kinder mit und ohne sonderpädagogischen Förderbedarf ausgewiesen werden. Gleichzeitig ,,...] bildet gerade die Forderung nach Standards von Inklusion im Grunde eine Kontradiktion gegenüber dem, was einen inklusiven Unterricht ausmacht, nämlichen einen Unterricht, der zwar Lernen entlang gemeinsamer Gegenstände organisiert, hierbei aber im Sinne der Individualisierung eine Vielfalt von Zugängen ermöglicht“ (Rödler 2012, S. 33).

Zusätzlich stehen evidenzbasierte Diagnose- und Förderprogramme (z. B. CMB oder DVB) nach Schumann (2016, S. 174) in der Tradition einer individualisierenden und defizitär ausgerichteten Diagnostik, die sich an einem medizinischen Modell orientiert. In diesen Modellen wird fast ausschließlich auf das Verhalten der Lernenden fokussiert, was zu einer Komplexitätsreduktion und Entkontextualisierung schulischer Lernprozesse führt, auf die ja gerade in der menschrechtlich orientierten Verortung von Inklusion und Partizipation als normorientierten sozialen Prozess hingewiesen wird. So werden auch im pädagogischen Bereich nahezu programmhaft Interventionen erforscht, wie z. B. im „Response to Intervention“ - (RTI) Modell 
(Limbach-Reich 2015). ${ }^{1}$ Nach Hinz wird bspw. ,,mit RTI [...] so getan als entwickelten sich Kinder im gesellschaftsfreien Raum [...]. Andere Barrieren für Lern- und Partizipationsprozesse als die dem Lernenden innewohnende kommen [...] nicht vor“" (Hinz 2016, S. 248). Willmann ergänzt diesbezüglich, dass eine inklusionsorientierte Didaktik hingegen ,auf das Erkennen und Beseitigen von Inklusionsbarrieren [zielt]. Nicht die Anpassung des Schülerverhaltens steht im Mittelpunkt der Interventionsmaßnahmen, sondern ganz im Gegenteil: die Anpassung der Schul- und Unterrichtsorganisation an den Entwicklungsstand und an die Bedürfnisse der einzelnen Schüler“ (Willmann 2015, S. 428). Der Einsatz evidenzbasierter Unterrichtsund Förderverfahren sowie eine datenbasierte Praxis als Grundlage für Förderentscheidungen und zur Beurteilung des Lernverlaufs der Kinder beschränken dabei den Blick einseitig auf individuumsorientierte Faktoren, wobei traditionelle Normalitätsvorstellung aufrechterhalten werden. So behandelt beispielsweise auch die klassische Testdiagnostik „Personen so, als wären sie Klons, die keine verschiedenen Ziele und Wünsche haben, sondern nur verschieden gut die Erwartung erfüllen, die Testmacher und seine Auftraggeber an ihn stellen. An einzelnen Menschen und ihren Fähigkeiten sind diese Testmacher nicht interessiert" (Lind 2020, S. 30), genauso wenig wie an den systemischen Bedingungen unter denen die geforderte Lernleistung zustande kommt. Gerade im Hinblick auf eine an Teilhabe ausgerichtete Bildung kann deshalb nicht darauf verzichtet werden, Tests so zu konstruieren, dass sie eine nachvollziehbare pädagogische Bedeutung haben und dabei helfen, die Bedingungen des Lernens in und an der Gemeinschaft zu verbessern (in Anlehnung an Lind 2020, S. 31). Innerhalb der Inklusionsforschung und auch der Grundschulforschung ist ein einseitiger Trend zu beobachten, der anhand quantitativ empirischer Methoden versucht, Evidenzen von Inklusion auszumachen, die jedoch von einer unspezifischen Verwendung der Begrifflichkeiten Partizipation und Inklusion zeugen, so dass es bspw. zu einem impliziten Primat von (kognitiver) Leistungsfähigkeit in der Beurteilung von Inklusion kommen kann (z.B. Heinrich und te Poel 2018). Bildung erfährt hier in ihrer Mehrdimensionalität eine Selbstentfremdung und wird auf einzelne vermeintlich evidenzrelevante Konstrukte reduziert. An einer evidenzbasierten Pädagogik kann man in diesem Fall monieren, dass das schulische Leistungsprinzip und die schulische Allokationsfunktion einer Heterogenitätsbejahung, wie sie für Partizipation und Inklusion gefordert wird, entgegenstehe (Sturm 2015; K. Müller 2018). Wie jedoch die Antagonismen der Leistungsgerechtigkeit und Inklusion pädagogisch - didaktisch verarbeitet werden könnten, kann evidenzbasiert bislang weder reflektiert oder untersucht werden (Müller und Pfrang 2021, i.E.).

\footnotetext{
${ }^{1}$ Der Begriff „Response-to-Intervention“ (RTI) bezeichnet einen Beschulungsansatz, der zum einen auf die Prävention von und die Integration bei sonderpädagogischem Förderbedarf abzielt und zum anderen eine alternative Form der Feststellung von Lern- und Entwicklungsbeeinträchtigungen darstellt (Gresham et al. 2005). Hauptanliegen ist es, unterrichtliche Maßnahmen so zu gestalten, dass jeder Lernende davon in ausreichendem Maß profitieren kann. Ob dies gelingt, wird auf Grundlage der Reaktionen der Kinder (Response) auf die pädagogischen Angebote (Intervention) bemessen, welche sich in der schulischen Leistungsentwicklung manifestieren. Kernmerkmale des RTI-Konzeptes sind die stufenweise aufgebauten Maßnahmen auf mehreren Förderebenen (Mehrebenenprävention), der Einsatz evidenzbasierter Unterrichts- und Förderverfahren sowie eine datenbasierte Praxis als Grundlage für Förderentscheidungen sowie zur Beurteilung des Lernverlaufs der Heranwachsenden. Diese Maßnahmen ermöglichen es den Lehrkräften, Lern- und Entwicklungsschwierigkeiten frühzeitig zu erkennen und angemessen darauf zu reagieren.
} 


\section{Ausblick: Inklusion und Partizipation als Aufgabe der Grundschulpädagogik}

Evidenzbasierte Forschung zu Inklusion und Partizipation in der Grundschulpädagogik einseitig zu kritisieren ist gleichermaßen naiv wie Evidenzbasierung zur Königsdisziplin in der Forschung zu erheben. Die genannten Diskussionspunkte zeigen auf, dass es verfehlt wäre, nur eine rein evidenzbasierte Grundschulpädagogik im Kontext von Inklusion und Partizipation zu betreiben. Wenn Grundschulpädagogik sich Inklusion und Partizipation als einem komplexen pädagogischen Wissenschafts- und Handlungsfeld nähern will, das unterschiedliche Lesarten von Inklusion und Partizipation zulässt, wird es in Gänze nahezu unmöglich, einzelne Faktoren so zu isolieren, so dass diese in ihren Effekten abgeschätzt werden können. Gleiches gilt für die Beforschung von Interaktionen, wenn Inklusion und Teilhabe als mehrdimensionale Lern- und Kommunikationsprozesse verstanden werden. Eine kritisch kulturwissenschaftliche Grundschulpädagogik muss also mehr können, als nur Effekte zu messen und sich den Modellen der pädagogischen Psychologie zu verpflichten (Willmann 2018, S. 110). Um die Komplexität des grundschulpädagogischen Handlungsfeldes in der Beforschung von Inklusion und Partizipation $\mathrm{zu}$ berücksichtigen, sind die Mehrdimensionalität, die Reziprozität und Widersprüchlichkeit dieses Forschungsfeldes multiparadigmatisch zu beleuchten. Von Bedeutung ist dann nicht nur der funktionelle Effekt einer Handlung, sondern die semantische Bedeutung, die diese Handlung in der Gruppe im Hinblick auf Inklusion und Partizipation gewinnt. Inklusion und Partizipation können so als komplexes sozial-ethisches Lernfeld verortet werden (Müller und Pfrang 2021 i.E.), dessen Gestaltung Pädagoginnen und Pädagogen unter Berücksichtigung der Mehrdimensionalität von Inklusion, Partizipation und Teilhabe als Aufgabe obliegt. Bildung entfaltet sich hier in ihrer sachbezogenen und sozialen Dimension (ebd.). Gerade für die Grundschulpädagogik mit dem Anspruch auf eine Schule für alle gilt es deshalb auch weiterhin neben dem individuellen Lernen das Lernen in und an der Gemeinschaft in der Forschung zu berücksichtigen. Inklusion, Partizipation und Teilhabe brauchen die Gemeinschaft, um sich als sozial-ethisches und individuelles Lernfeld entfalten zu können. Eine rein evidenzbasierte Grundschulforschung zu Inklusion und Partizipation vermag zwar Wirksamkeitsnachweise einzelner, inklusionsorientierter Parameter oder spezifischer Fördermethoden und -ansätze nachzuweisen, trägt aber durch eine starke Fokussierung auf ebendiese Parameter oder individuell-kognitive Lernprozesse auch zu einer „Entkulturierung des Lernens“ (Rödler 2016) bei, da für die soziale Dimension von Bildung bedeutsame Lern- und Kommunikationsprozesse aus dem Blick geraten könnten.

Inklusion und Partizipation verweisen hier auf den Anspruch grundlegender Bildung, sich im Spannungsfeld der Vergesellschaftung und Individualisierung normorientiert bewegen zu können. Für Wissenschaft und Praxis bieten Inklusion und Partizipation also normorientierte Orientierungen. Entsprechend ist dieser normorientierte Anspruch sowohl an die Forschung als auch an die grundsätzlichen Überlegungen zu Inklusion und Partizipation als mehrdimensionales komplexes pädagogisches Handlungsfeld zurückzubinden. Neben den Zugriffsmöglichkeiten auf Bildungsangebote müssen auch die subjektiven Fähigkeiten und volitionalen Vor- 
aussetzungen in den Blick genommen werden, die es einer Person ermöglichen, die zur Verfügung stehenden Bildungsangebote für ihre Bildungsprozesse zu nutzen (Horster 2011, S. 16). Die normorientierte pädagogische Vermittlung zwischen individuellen Lernvoraussetzungen und systemischen Bedingungen und Strukturen wird zur zentralen pädagogisch - didaktischen Aufgabe (Müller und Pfrang 2021, i.E.). Es bleibt also weiterhin eine wichtige Aufgabe der Grundschulpädagogik das Ziel der allgemeinen Grundbildung für alle an der Schnittstelle zwischen empirischer Bildungsforschung, interdisziplinärer Expertise zu Kindheitsforschung und Didaktik sowie bildungspolitischer Planung und Steuerung zu behandeln und die sich in der Grundschule darstellende Praxis im Hinblick auf Inklusion und Partizipation zu reflektieren, theoretisieren und weiterzuentwickeln.

Funding Open Access funding enabled and organized by Projekt DEAL.

Open Access Dieser Artikel wird unter der Creative Commons Namensnennung 4.0 International Lizenz veröffentlicht, welche die Nutzung, Vervielfältigung, Bearbeitung, Verbreitung und Wiedergabe in jeglichem Medium und Format erlaubt, sofern Sie den/die ursprünglichen Autor(en) und die Quelle ordnungsgemäß nennen, einen Link zur Creative Commons Lizenz beifügen und angeben, ob Änderungen vorgenommen wurden.

Die in diesem Artikel enthaltenen Bilder und sonstiges Drittmaterial unterliegen ebenfalls der genannten Creative Commons Lizenz, sofern sich aus der Abbildungslegende nichts anderes ergibt. Sofern das betreffende Material nicht unter der genannten Creative Commons Lizenz steht und die betreffende Handlung nicht nach gesetzlichen Vorschriften erlaubt ist, ist für die oben aufgeführten Weiterverwendungen des Materials die Einwilligung des jeweiligen Rechteinhabers einzuholen.

Weitere Details zur Lizenz entnehmen Sie bitte der Lizenzinformation auf http://creativecommons.org/ licenses/by/4.0/deed.de.

\section{Literatur}

Altrichter, H., \& Maag Merki, K. (2010). Steuerung der Entwicklung des Schulwesens. In H. Altrichter \& K. Maag Merki (Hrsg.), Handbuch Neue Steuerung im Schulsystem (S. 1-28). Wiesbaden: VS.

Betz, T., Bischoff-Pabst, S., Eunicke, N., \& Menzel, B. (2019). Kinder zwischen Chancen und Barrieren. Forschungsbericht 2. Zum Verhältnis von Schule und Familie aus der Sicht von Kindern: ihre Perspektiven, ihre Positionen. Gütersloh: Bertelsmann.

Biewer, G. (2010). Grundlagen der Heilpädagogik und Inklusiven Pädagogik (2. Aufl.). Bad Heilbrunn: Klinkhardt.

Boban, I., \& Hinz, A. (2019). ,Kritischer Sachunterricht“ auf der Basis von Paulo Freires ,Pedagógica crítica - eine inklusive, demokratische Art ,die Welt' zu lesen? In M. Siebach, J. Simon \& T. Simon (Hrsg.), Ich und Welt verknüpfen. Allgemeinbildung, Vielperspektivität, Partizipation und Inklusion im Sachunterricht (S. 77-88). Baltmannsweiler: Schneider.

Buchhaas-Birkholz, D. (2009). Die >empirische Wende< in der Bildungspolitik und in der Bildungsforschung. Zum Paradigmenwechsel des BMBF im Bereich der Forschungsförderung. Erziehungswissenschaft, 39, 27-33.

Danz, S. (2020). Partizipation meint Teilhabe und Solidarität. Visionen für eine bessere Zukunft für alle. In S. Gerhartz-Reiter \& C. Reisenauer (Hrsg.), Partizipation und Schule. Perspektiven auf Teilhabe und Mitbestimmung von Kindern und Jugendlichen (S. 63-78). Wiesbaden: Springer VS.

Dietrich, C. (2017). Teilhaben - Teil sein - Anteil nehmen. In I. Miethe, A. Tervooren \& N. Ricken (Hrsg.), Bildung und Teilhabe. Zwischen Inklusionsforderung und Exklusionsdrohung (S. 29-46). Wiesbaden: Springer VS.

Dormann, C., Binnewies, C., Koch, A., van Ackeren, I., Clausen, M., Preisendörfer, P., Schmidt, U., \& Zlatkin-Troitschanskaia, O. (2016). Transferring best evidence into practice: assessment of evidencebased school management. Journal for Educational Research Online, 8(3), 14-38. 
Fend, H. (2006). Neue Theorie der Schule. Einführung in das Verstehen von Bildungssystemen. Wiesbaden: VS.

Gebauer, M., \& Simon, T. (2012). Inklusiver Sachunterricht konkret: Chancen, Grenzen, Perspektiven. Widerstreit Sachunterricht. http://www.widerstreit-sachunterricht.de/ebenel/superworte/inkluaion/ gebauer_simon.pdf. Zugegriffen: 17. Febr. 2021.

Götz, M., Miller, S., Einsiedler, W., \& Vogt, M. (2019). Diskussionspapier zum Selbstverständnis der Grundschulpädagogik als wissenschaftliche Disziplin. In C. Donie, F. Foerster, M. Obermayr, A. Deckwerth, G. Kammermeyer, G. Lenske, M. Leuchter \& A. Wildemann (Hrsg.), Grundschulpädagogik zwischen Wissenschaft und Transfer (S. 12-21). Wiesbaden: Springer VS.

Gräsel, C. (2010). Stichwort: Transfer und Transferforschung im Bildungsbereich. Zeitschrift für Erziehungswissenschaft, 13(1), 7-20.

Gresham, F. M., Van Der Heyden, A., \& Witt, J.C. (2005). Response to intervention in the identification of learning disabilities: empirical support and future challenges. http://www.joewitt.org/Downloads/ Response\%20to\%20Intervention\%20MS\%20Gresham\%20\%20Vanderheyden\%20Witt.pdf. Zugegriffen: 28. Febr. 2021.

Heinrich, M., \& Te Poel, K. (2018). Integration durch Leistung als „Inklusionsfalle“. Governanceanalytische Konsequenzen eines nicht-inklusiven Bildungsmonitorings zur Evaluation der Umsetzung der UN-BRK. In M. Walm, T. Häcker, F. Radisch \& A. Krüger (Hrsg.), Empirisch-pädagogische Forschung in inklusiven Zeiten. Konzeptualisierung, Professionalisierung, Systementwicklung (S. 253-268). Bad Heilbrunn: Klinkhardt.

Herzog, W. (2019). Kritik der evidenzbasierten Pädagogik. Lehren und Lernen, 45(5), 21-28.

Hildebrandt, E., \& Maischatz, K. (2020). Partizipation im Unterricht. In N. Skorsetz, M. Bonanati \& D. Kucharz (Hrsg.), Diversität und soziale Ungleichheit. Herausforderungen an die Integrationsleistung der Grundschule (S. 164-168). Wiesbaden: Springer VS.

Hinz, A. (2016). Response-to-Intervention - eine Scheinlösung für die Herausforderung inklusionsorientierter Diagnostik?! In B. Amrhein (Hrsg.), Diagnostik im Kontext inklusiver Bildung. Theorien, Ambivalenzen, Akteure, Konzepte (S. 243-257). Bad Heilbrunn: Klinkhardt.

Horster, D. (2011). Bildungsgerechtigkeit aus sozialphilosophischer Sicht. In T. Meyer \& U. Vorholt (Hrsg.), Bildungsgerechtigkeit als politische Aufgabe. Dortmunder politisch-philosophische Diskurse (S. 11-20). Bochum: Projektverlag.

Konsortium der Studie zur Entwicklung von Ganztagsschulen (StEG) (Hrsg.). (2019). Individuelle Förderung: Potenziale der Ganztagsschule. Frankfurt am Main: Das Konsortium der Studie zur Entwicklung von Ganztagsschulen (StEG) 2019. https://www.pedocs.de/frontdoor.php?source_opus=19109. Zugegriffen: 9. Okt. 2020.

Kruschel, R. (Hrsg.). (2017). Menschenrechtsbasierte Bildung. Inklusive und demokratische Lern- und Erfahrungswelten im Fokus. Bad Heilbrunn: Klinkhardt.

Leßmann, B. (2020). Autorenrunden. Kinder entwickeln literale Kompetenzen. Eine interdisziplinäre theoriebildende Studie zu Gesprächen über eigene Texte in der Grundschule. Münster: Waxmann.

Limbach-Reich, A. (2015). „Response to Intervention“ (RTI) im Spannungsfeld Inklusiver Diagnostik. In H. Schäfer \& C. Rittmeyer (Hrsg.), Inklusive Diagnostik (S. 478-499). Weinheim: Beltz.

Lind, G. (2020). Messen psychometrische Tests, was sie vorgeben zu messen? Die Bildungsforschung und die Krise der psychometrischen Tests. Lehren und Lernen, 46(6), 29-32.

Ministerium für Soziales und Integration (Hrsg.). (2015). Aktionsplan der Landesregierung zur Umsetzung der UN-Behindertenrechtskonvention in Baden-Württemberg. https://sozialministerium. baden-wuerttemberg.de/fileadmin/redaktion/msm/intern/downloads/Downloads_Menschen_mit_ Behinderungen/Aktionsplan_UN-BRK_Aug-2016_barrierefrei.pdf. Zugegriffen: 25. Febr. 2021.

Moser, V. (2019). Inklusion zwischen normativer Orientierung und Evidenzbasierung. In M. Hartmann, M. Hummel, M. Lichtblau, J. M. Löser \& S. Thoms (Hrsg.), Facetten inklusiver Bildung.: Nationale und internationale Perspektiven auf die Entwicklung inklusiver Bildungssysteme (S. 31-39). Bad Heilbrunn: Klinkhardt.

Müller, F. J. (2018). Blick zurück nach vorn - WegbereiterInnen der Inklusion. Bd. 1 + 2. Gießen: Psychosozial-Verlag.

Müller, K. (2018). Leistungsgerechtigkeit im Kontext von Inklusion. In T. Sansour, J. Riegert \& O. Musenberg (Hrsg.), Bildung und Leistung. Differenz zwischen Selektion und Anerkennung (S. 94-103). Bad Heilbrunn: Klinkhardt.

Müller, K., \& Pfrang, A. (2021 i.E.). Sozial-ethische Vielfalt als Herausforderung an die allgemeine Didaktik. In T. Iwers \& U. Graf (Hrsg.), Vielfalt thematisieren - Gemeinsamkeiten und Unterschiede gestalten. Herausforderungen und Chancen in pädagogischen Kontexten (S. 62-72). Bad Heilbrunn: Klinkhardt. 
Pape, M. (2016). Didaktisches Handeln in jahrgangsheterogenen Grundschulklassen. Eine qualitative Studie zur Inneren Differenzierung und zur Anleitung des Lernens. Bad Heilbrunn: Klinkhardt.

Pech, D., Schomaker, C., \& Simon, T. (Hrsg.). (2019). Forschungen zur Didaktik des Sachunterrrichts. Bad Heilbrunn: Klinkhardt.

Prengel, A. (2006). Pädagogik der Vielfalt. Verschiedenheit und Gleichberechtigung in Interkultureller, Feministischer und Integrativer Pädagogik. Wiesbaden: Springer VS.

Radtke, F. O. (2013). Die Regierungskunst der Betriebswirte. Eine Zwischenbilanz der $>$ neuen Steuerung < des Bildungssystems. Der Pädagogische Blick. Zeitschrift für Wissenschaft und Praxis in pädagogischen Berufen (3). https://docplayer.org/36994729-Die-regierungskunst-der-betriebswirte.html. Zugegriffen: 8. Febr. 2021.

Rödler, P. (2012). Inklusion und Standards - eine verständliche, aber paradoxe Forderung. gemeinsam lernen, 1, 33-40.

Rödler, P. (2013). Inklusion ist evident begründbar, aber nicht evident machbar! - Das Problem pädagogischer Praxis mit Ergebnissen > evidenzbasierter< Wissenschaft. Behindertenpädagogik, 52(4), $381-388$.

Rödler, P. (2016). RTI - ein Konzept der Entkulturierung von Lernen. In B. Amrhein (Hrsg.), Diagnostik im Kontext inklusiver Bildung. Theorien, Ambivalenzen, Akteure, Konzepte (S. 232-241). Bad Heilbrunn: Klinkhardt.

Rommelspacher, B. (1995). Dominanzkultur: Texte zur Fremdheit und Macht. Berlin: Orlanda Frauenverlag.

Rommelspacher, B. (2002). Anerkennung und Ausgrenzung. Deutschland als multikulturelle Gesellschaft. Frankfurt a. Main/New York: Campus Verlag.

Schönwiese, V. (2016). Behindertenbewegung. In I. Hedderich, G. Biewer, J. Hollenweger \& R. Markowetz (Hrsg.), Handbuch Inklusion und Sonderpädagogik (S. 44-48). Bad Heilbrunn: Klinkhardt.

Schrader, J. (2014). Analyse und Förderung effektiver Lehr-Lernprozesse unter dem Anspruch evidenzbasierter Bildungsreform. Zeitschrift für Erziehungswissenschaft, 17(2), 193-223.

Schumann, B. (2016). „Auf dem Weg zur Inklusion“ oder zur „Sonderpädagogisierung“ der allgemeinen Schule? In B. Amrhein (Hrsg.), Diagnostik im Kontext inklusiver Bildung. Theorien, Ambivalenzen, Akteure, Konzepte (S. 168-177). Bad Heilbrunn: Klinkhardt.

Schwab, S. (2016). Partizipation. In I. Hedderich, G. Biewer, J. Hollenweger \& R. Markowetz (Hrsg.), Handbuch Inklusion und Sonderpädagogik (S. 127-131). Bad Heilbrunn: Klinkhardt.

Seitz, S. (2020). Dimensionen inklusiver Didaktik - Personalität, Sozialität und Komplexität. Zeitschrift für Inklusion (2). https://www.inklusion-online.net/index.php/inklusion-online/article/view/570. Zugegriffen: 8. Febr. 2021.

Sturm, T. (2015). Inklusion: Kritik und Herausforderung des schulischen Leistungsprinzips. Erziehungswissenschaft, 26(51), 25-32.

Tenorth, H.-E. (2014). Evidenzbasierte Bildungsforschung vs. Pädagogik als Kulturwissenschaft - Über einen neuerlichen Paradigmenstreit in der wissenschaftlichen Pädagogik. Neveléstudomány Tanulmányok 3, S. 5-21. http://nevelestudomany.elte.hu/downloads/2014/nevelestudomany_2014_3_521.pdf. Zugegriffen: 8. Febr. 2021.

Tooley, J., \& Darby, D. (1998). Educational research: a critique. https://www.voced.edu.au/content/ngv: 1563. Zugegriffen: 8. Febr. 2021.

UN-Behindertenrechtskonvention (UN-BRK) (2006). Übereinkommen über die Rechte von Menschen mit Behinderungen. http://www.behindertenbeauftragte.de/SharedDocs/Publikationen/UN_Konvention_ deutsch.pdf;jsessionid=51439F4E442B0A94799F736EBA20E4DA.2_cid355?_blob=publication File\&v=2. Zugegriffen: 24. Juli 2017.

Uphoff, I. K. (2009). Die Professionalisierung des Lehrerberufs - oder: Vom pädagogischen Umgang mit Auffälligkeiten. In A. Nießeler \& I. K. Uphoff (Hrsg.), Pädagogische Auffälligkeiten: Deutungsmuster von Verhaltensstörungen und Verhaltensauffälligkeiten kritisch betrachtet (S. 63-76). Würzburg: Königshausen \& Neumann.

Weick, K. E. (1976). Educational organizations as loosely coupled systems. Administrative Science Quarterly, 21(1), 1-19.

Wiesemann, J., \& Schreyer, H. (2008). Zur Bedeutung von Differenz im Lernalltag der Kinder einer JÜLKlasse. Ergebnisse einer Beobachtungsstudie. In J. Ramseger \& M. Wagener (Hrsg.), Chancenungleichheit in der Grundschule. Ursachen und Wege aus der Krise (S. 159-162). Wiesbaden: VS.

Willmann, M. (2015). Emotional-soziale Schwierigkeiten und Verhaltensstörungen: Diagnostik und Assessment in der inklusiven Schule. In H. Schäfer \& C. Rittmeyer (Hrsg.), Handbuch inklusive Diagnostik (S. 419-432). Weinheim: Beltz. 
Willmann, M. (2018). Vermessung des Verhaltens, Normierung zur Inklusion? RTI als evidenzbasierte Pädagogik - eine Kritik. Zeitschrift für Grundschulforschung, 11(1), 101-114.

Zlatkin-Troitschanskaia, O., Förster, M., \& Preuße, D. (2012). Implementierung und Wirksamkeit der erweiterten Autonomie im öffentlichen Schulwesen - Eine Mehrebenbetrachtung. In A. Wacker, U. Maier \& J. Wissinger (Hrsg.), Schul- und Unterrichtsreform durch ergebnisorientierte Steuerung - Empirische Befunde und forschungsmethodische Implikationen (S. 79-108). Wiesbaden: Springer VS. 